\title{
Avaliação de métodos radiográficos utilizados na localização vertical de sítios eleitos para instalação de mini-implantes
}

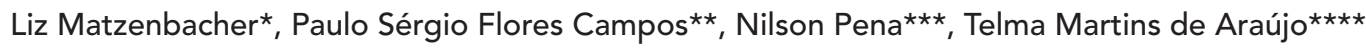

\section{Resumo}

Objetivo: avaliar a efetividade de métodos de diagnóstico por imagem utilizados na localização vertical de sítios eleitos para inserção de mini-implantes. Metodologia: a amostra foi composta de quatro pacientes, nos quais foram estabelecidos 32 sítios inter-radiculares, na região posterior, para instalação de mini-implantes, sítios estes representados por orifícios preenchidos com guta-percha em moldeiras de acetato (PCg - ponto de contato das coroas dentárias na moldeira de acetato; PIg - ponto de inserção do mini-implante na moldeira de acetato). Foram feitas radiografias periapicais, interproximais e tomografias computadorizadas de feixe cônico, com as moldeiras posicionadas em boca, sendo considerados os seguintes pontos: PC - imagem radiodensa do ponto PCg; PI - imagem radiodensa do ponto PIg; PCX - ponto de contato entre as coroas dentárias, determinado sobre a radiografia. Medidas verticais utilizadas: padrão-ouro - de PCg a PIg; medida 1 - de PC a PI; e medida 2 - de PCx a PI. As medidas foram comparadas através de análise descritiva e do teste $t$ de Student. Resultados: para a medida 1, foi observada diferença estatisticamente significante, em relação ao padrão-ouro, em 4,1\%, 25\% e 100\% das medidas avaliadas nas tomografias computadorizadas, radiografias interproximais e periapicais, respectivamente. Para a medida 2 , foi observada diferença estatisticamente significante, em relação ao padrão-ouro, em 4,1\%, 56,2\% e 100\% das medidas avaliadas nas tomografias computadorizadas, radiografias interproximais e periapicais, respectivamente Conclusões: a tomografia computadorizada de feixe cônico foi o exame mais preciso na avaliação da posição vertical dos sítios eleitos para instalação de mini-implantes; a radiografia interproximal pode, com reserva, ser utilizada; a radiografia periapical apresentou resultados insatisfatórios, sendo contra-indicada para este fim.

Palavras-chave: Diagnóstico por imagem. Radiografia dentária.

Tomografia computadorizada por raios-X. Procedimentos de ancoragem ortodôntica.

* Aluna do Curso de Especialização em Ortodontia do Centro de Ortodontia e Ortopedia Facial da Faculdade de Odontologia da Universidade Federal da Bahia - FO.UFBA.

** Professor Associado Livre-Docente de Radiologia da Faculdade de Odontologia da Universidade Federal da Bahia.

*** Doutor em Radiologia pela Faculdade de Odontologia de Piracicaba (UNICAMP - Universidade Estadual de Campinas). Pesquisador da FAPESB (Fundação de Amparo à Pesquisa do Estado da Bahia).
Doutora e Mestre em Ortodontia pela UFRJ. Professora Titular de Ortodontia da FO.UFBA. Coordenadora do Curso de Especialização em OrtoDoutora e Mestre em Ortodontia pela UFRJ. Professora Titular de Ortodontia da FO.
dontia da FO.UFBA. Diretora do Board Brasileiro de Ortodontia e Ortopedia Facial. 


\section{INTRODUÇÃO}

Os mini-implantes requerem intervenção cirúrgica simples e rápida ${ }^{5,12}$, porém, para que sejam instalados com segurança, é importante uma cuidadosa avaliação clínica e radiográfica, um adequado planejamento e um protocolo de instalação cuidadoso, com especial atenção à anatomia da região, eliminando riscos de lesão a estruturas nobres ${ }^{2,3}$.

Os mini-implantes podem ser instalados em regiões anatômicas com quantidade mínima de osso, sendo que o espaço inter-radicular é freqüentemente escolhido. Se a quantidade de osso interproximal e a proximidade das raízes forem incorretamente avaliadas, há risco de perfuração radicular ${ }^{7,17-23,26,28}$. Estudos comprovam que o ligeiro contato do dispositivo com o ligamento periodontal ou cemento, sem comprometimento do feixe vásculo-nervoso ou invasão do canal, não afeta a vitalidade dos dentes. Entretanto, todos os cuidados fazem-se necessários para evitar este tipo de intercorrência, devido ao desconforto para o paciente e implicações clínicas e legais que podem ser geradas por esta complicaçãa ${ }^{2,21}$.

Além disso, a proximidade entre o mini-implante e a raiz do dente é o maior fator de insucesso desses dispositivos. A proximidade da lâmina dura, por exemplo, pode comprometer a estabilidade do mini-implante ${ }^{16}$. Quando o espaço inter-radicular é reduzido, deve-se selecionar mini-implantes com diâmetro menor, sendo recomendável o de, no mínimo, $1,4 \mathrm{~mm}$, pois os menores apresentam maior risco de fratura quando da instalação ou remoção ${ }^{1}$.

Defende-se que a identificação precisa do local eleito pode ser feita através de imagens dos guias cirúrgicos $3,13,15,20,28$. Porém, sabe-se que imagens bidimensionais obtidas através das radiografias intrabucais não refletem, necessariamente, a verdadeira relação do espaço com as estruturas anatômicas adjacentes, pois objetos em planos diferentes podem apresentar projeções oblíquas e imagens distorcidas ${ }^{6,19}$. Kim et al. ${ }^{13}$ e Kitai et al. ${ }^{14}$ sugeriram a utilização de tomografia computadorizada para visualização de guias cirúrgicos. Entretanto, apesar de apresentar imagens tridimensionais, a tomografia computadorizada tem um custo relativamente alto e expõe o paciente a uma dose de radiação mais elevada, quando comparada às radiografias intrabucais.

A evolução dos mini-implantes não deve se restringir apenas aos diferentes tipos, formas e técnicas operatórias, devendo também estar voltada para o desenvolvimento dos meios de diagnóstico por imagem ${ }^{8,11}$. Assim, justifica-se o estudo sobre o grau de precisão dos exames radiográficos, com a finalidade de melhor aproveitá-los como método seguro para o planejamento dos mini-implantes.

Sabendo-se, portanto, que a correta eleição do ponto de inserção do mini-implante é de fundamental importância para o sucesso do procedimento cirúrgico, e que o espaço inter-radicular é, não raro, restrito, justifica-se a preocupação a respeito da técnica radiográfica que melhor informe o ponto de inserção supra-referido.

\section{MATERIAL E MÉTODOS}

Foram selecionados quatro indivíduos, do gênero feminino, na faixa etária de 25 a 28 anos, pacientes do Centro de Ortodontia e Ortopedia Facial Prof. José Édimo Soares Martins da Faculdade de Odontologia da Universidade Federal da Bahia. Esta seleção baseou-se nos seguintes critérios: dentadura permanente completa até os primeiros molares, ausência de apinhamento posterior e necessidade de tomografia computadorizada para planejamento de instalação de mini-implantes para ancoragem.

Para realização das radiografias e das tomografias, os pacientes utilizaram moldeiras de acetato, onde os sítios eleitos para instalação de mini-implantes foram representados por orifícios preenchidos com guta-percha. Para tanto, foram confeccionados modelos em gesso dos arcos dentários de cada paciente e, a partir destes, confeccionadas moldeiras em plastificadora a vácuo Plastvac - P7 
(Bio-art, São Carlos/SP, Brasil), utilizando placas de acetato de espessura de $1 \mathrm{~mm}$ (Whiteness FGM, Joinville/SC, Brasil).

Foram eleitos locais para instalação de miniimplantes nos espaços inter-radiculares entre o primeiro pré-molar e o segundo pré-molar e entre o segundo pré-molar e o primeiro molar de todos os hemiarcos, totalizando 32 sítios. $\mathrm{O}$ ponto de inserção foi estabelecido clinicamente na intersecção de uma linha imaginária vertical que passava pelo ponto de contato e a zona de transição entre a mucosa ceratinizada e a mucosa livre. As distâncias entre os pontos de contato e os pontos de inserção foram aferidas clinicamente com uma sonda milimetrada e transferidas, em seguida, para as moldeiras de acetato (Fig. 1A,C). Para colocação dos marcadores, as moldeiras foram perfuradas com uma ponta diamantada $n^{\circ} 2200$ (Fig. 1B, D), produzindo orifícios de $1 \mathrm{~mm}$ de diâmetro, que foram preenchidos com guta-percha.

Os pacientes foram submetidos a três técnicas de diagnóstico por imagem: radiografias interproximais e periapicais das regiões posteriores da maxila e da mandíbula e tomografia computadorizada de feixe cônico. Os exames foram executados
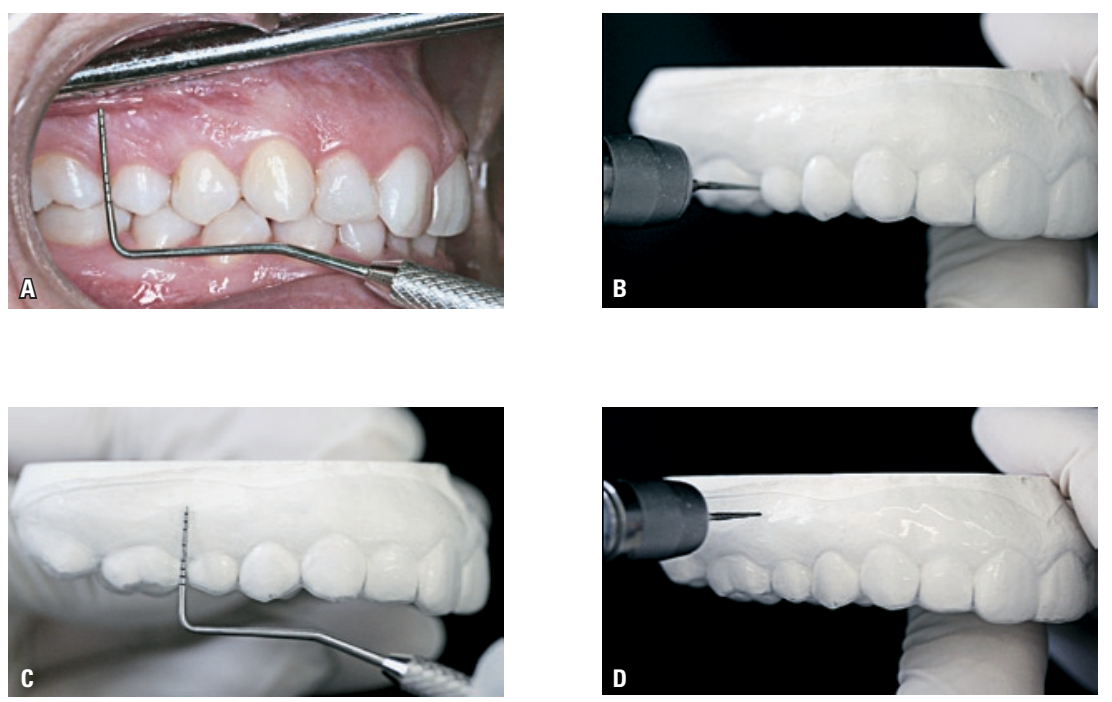

FIGURA 1 - A) Eleição do local para instalação dos mini-implantes, no limite entre as mucosas livre e ceratinizada. B) Perfuração do ponto de contato (broca $\left.n^{\circ} 2200\right)$.

C) Transferência do ponto eleito para a moldeira de acetato. D) Perfuração do ponto de inserção (broca nº 2200).

com as moldeiras de acetato posicionadas na boca do paciente (Fig. 2).

\section{Obtenção das radiografias interproximais e periapicais}

As radiografias interproximais e periapicais foram obtidas com filmes da marca Kodak, modelo InSight (Rochester, Nova York, EUA). Foi adotada a técnica da bissetriz com suporte Prisma (Prisma Produtos Clínicos Ltda, Brasília/DF, Brasil), sendo utilizado um aparelho periapical Heliodent Vario (Sirona - The Dental Company, Bensheim, Alemanha), com ampola ajustada para operar com tensão de $70 \mathrm{KVp}$, corrente de $7 \mathrm{~mA}$, tempo de exposição em 0,4 segundos e distância área focalfilme de $27 \mathrm{~cm}$ e $30 \mathrm{~cm}$ para as radiografias interproximais e periapicais, respectivamente. $\mathrm{O}$ método de processamento empregado foi o automático, com processadora Periomat Plus (Dürr Dental, Bietigheim-Bissingen, Alemanha) regulada para operar com tempo, de seco a seco, de 5 minutos.

Foram realizadas duas radiografias interproximais e quatro periapicais de cada paciente, para a região posterior (superiores e inferiores, lados direito e esquerdo).

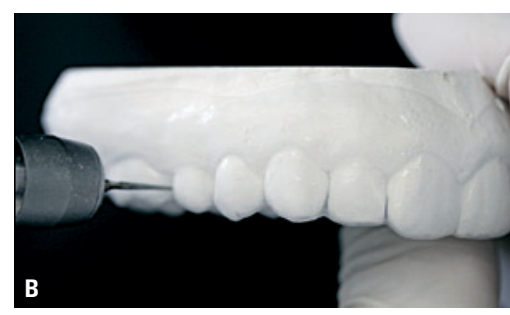



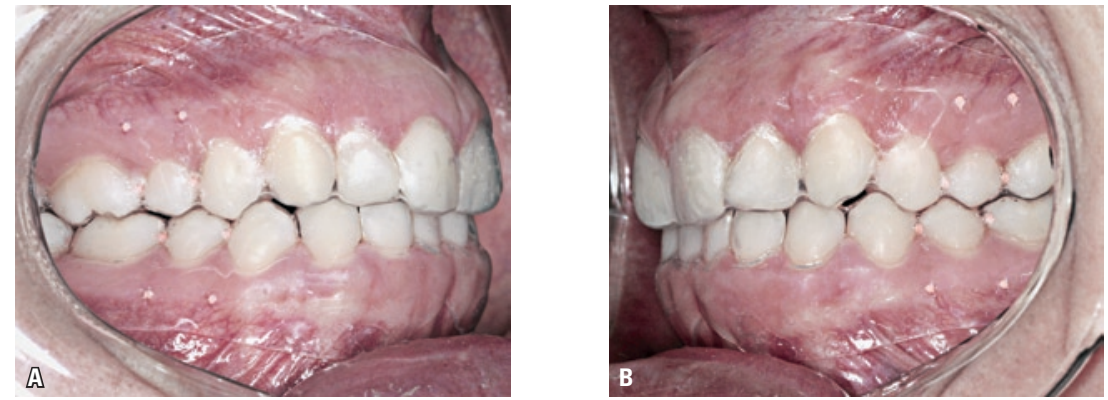

FIGURA 2 - Moldeiras de acetato posicionadas na boca, com os pontos selecionados e preenchidos com guta-percha: ponto de contato entre as coroas dentárias e local eleito para inserção de mini-implantes.

\section{Obtenção das tomografias computadorizadas}

As tomografias computadorizadas de feixe cônico foram realizadas em um aparelho i-CAT Cone Beam 3-D Dental Imaging System (Imaging Sciences Internacional, Hatfield, PA, EUA), que teve sua ampola ajustada para operar com uma tensão de $120 \mathrm{KVp}$ e corrente de $46,72 \mathrm{~mA}$. O protocolo de aquisição adotado foi o de dois arcos, com FOV de $8 \mathrm{~cm}$ e voxel de $0,2 \mathrm{~mm}$ ( $2 \mathrm{arc}, 8 \mathrm{~cm}$, 40sec, 0,2 voxel, max. res.). A aquisição, reconstrução e análise das imagens, foram executadas com auxílio do software XoranCat versão 3.0.34 (Xoran Technologies, Ann Arbor, Michigan), por um especialista em Radiologia com experiência em tomografia computadorizada de feixe cônico.

\section{Pontos e medidas}

Para avaliação, foram utilizados cinco pontos, a saber:

PCg - ponto de contato das coroas dentárias na moldeira de acetato, preenchido com guta-percha (Fig. 3A);

PIg - ponto de inserção do mini-implante na moldeira de acetato, preenchido com guta-percha (Fig. 3A);

PC - imagem radiopaca do ponto PCg nas radiografias e na tomografia computadorizada (Fig. 3B, C, D; 5B);

PI - imagem radiopaca do ponto PIg nas radiografias e na tomografia computadorizada (Fig. 3B, C, D);
PCx - ponto de contado entre as coroas dentárias, determinado sobre a radiografia pelo examinador (Fig. 3C, D; 5B). Este ponto foi criado para identificar o deslocamento dos pontos PCg e PIg nas imagens radiográficas.

Para o estudo, foram obtidas duas medidas lineares nas imagens radiográficas: medida $1-$ do ponto PC ao ponto PI; medida 2 - do ponto PCX ao ponto PI. As mensurações feitas diretamente sobre as moldeiras de acetato foram consideradas o padrão-ouro: do ponto PCg ao ponto PIg.

As distâncias entre os pontos, tanto nas radiografias quanto nas moldeiras de acetato, foram aferidas com paquímetro digital de alta precisão Mitutoyo Digimatic Caliper 0,01-150mm/0,0005"-6" (Mitutoyo, Indústria Brasileira) por um único examinador (Fig. 4A, C). Esse procedimento foi repetido, para testar e validar os resultados, por mais duas vezes, com uma semana de intervalo.

Como os pontos da moldeira de acetato apresentavam diâmetro de $1,0 \mathrm{~mm}$, considerou-se o centro do ponto para realizar as mensurações (Fig. 4A).

As mensurações sobre as radiografias interproximais e periapicais foram realizadas com o auxílio de negatoscópio, em sala escurecida, com máscara de papel-cartão preto e janela de tamanho correspondente ao do filme radiográfico. Os pontos PCx foram marcados com lapiseira $0,3 \mathrm{~mm}$ (Pentel, Japão) sobre um filme de acetato com $0,003 \mathrm{~mm}$ de espessura para traçado cefalométrico (3M - Unitek, Monrovia, EUA), fixado 

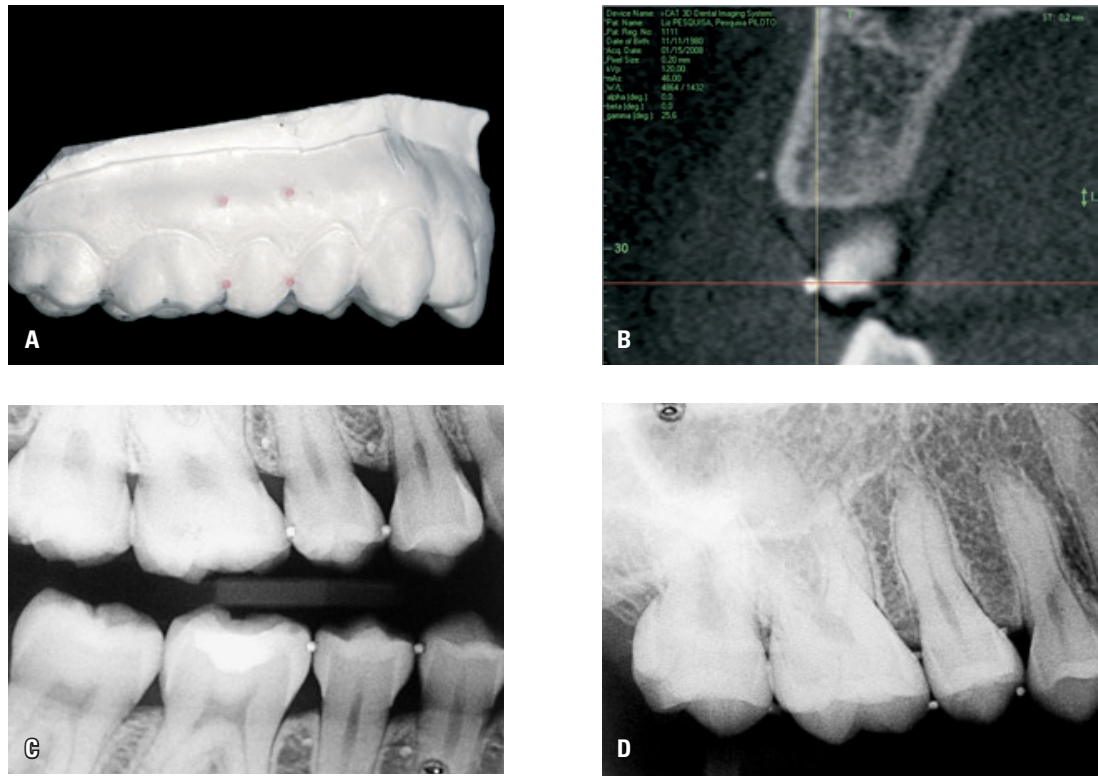

FIGURA 3 - llustração dos pontos utilizados no estudo: A) moldeira de acetato; B) tomografia computadorizada, plano coronal; C) radiografia interproximal; D) radiografia periapical.
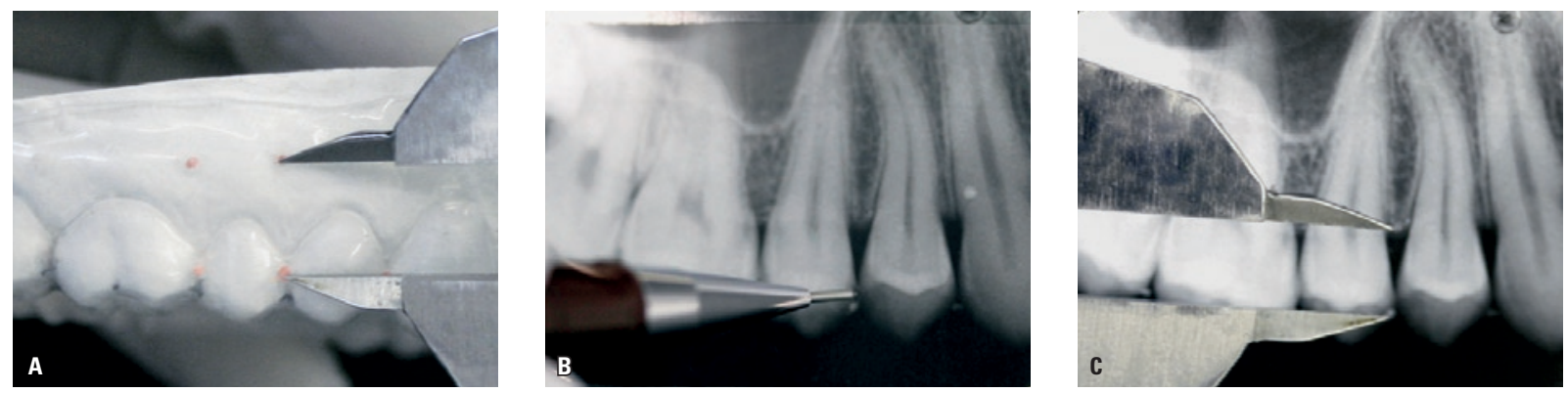

FIGURA 4 - A) Mensuração na moldeira de acetato. B) Marcação do ponto PCx. C) Mensuração na radiografia periapical com paquímetro digital.

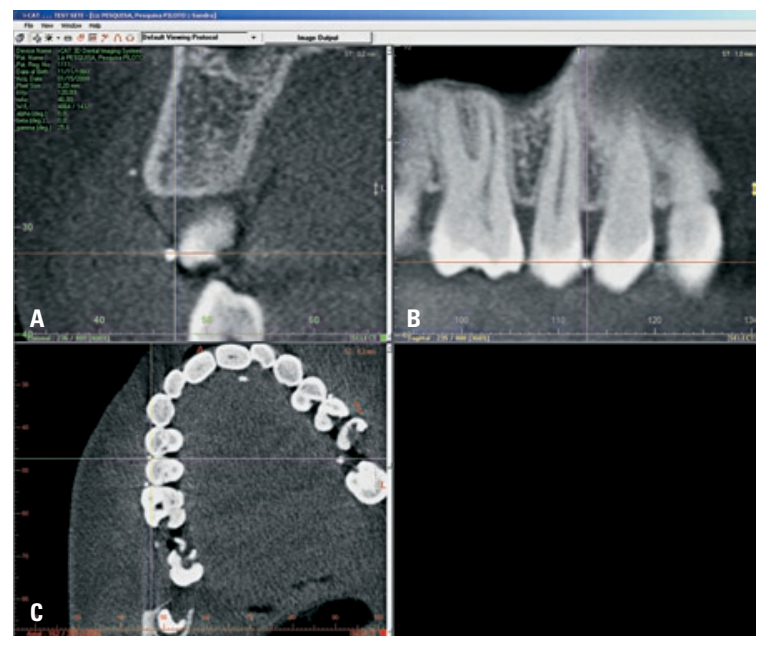

FIGURA 5 - Imagem do ambiente de avaliação multiplanar do software XoranCat, demonstrando a análise da região entre pré-molares do lado direito: A) corte coronal, onde se pode visualizar os pontos $\mathrm{PC}$ e Pl; B) corte sagital, identificação do ponto PCx, sobreposto ao PC; C) corte axial, utilizado para auxiliar o posicionamento dos demais cortes. 

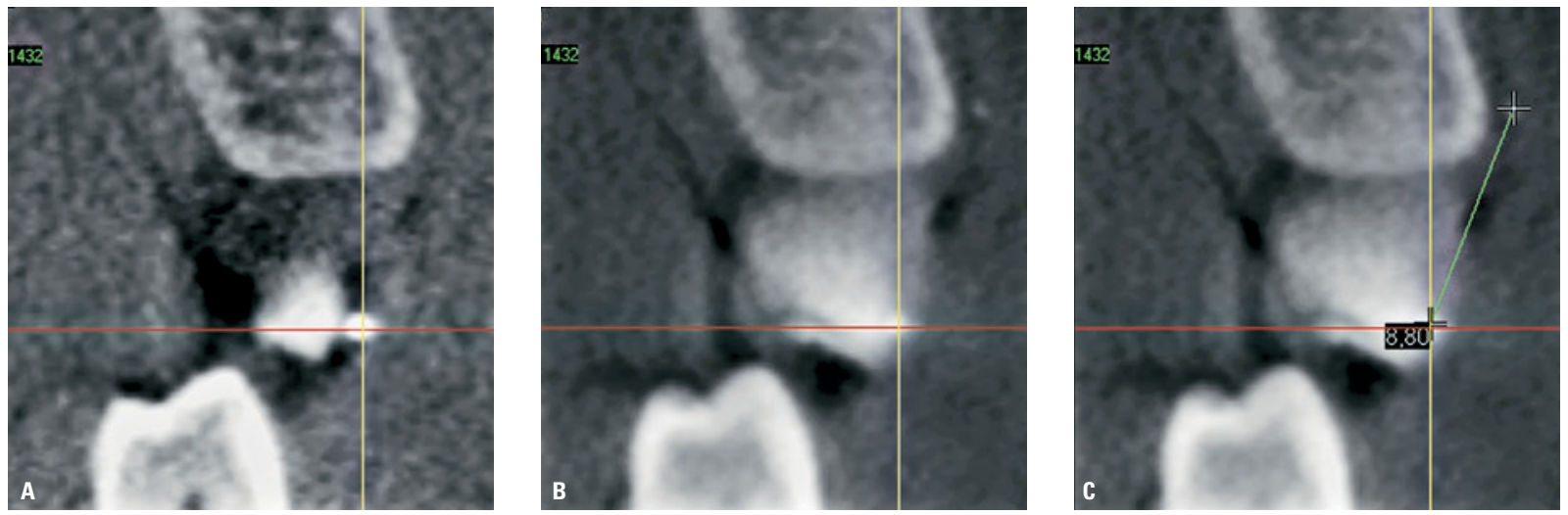

FIGURA 6 - A) Imagem de um corte da tomografia computadorizada com espessura de 0,2mm, identificando o ponto PC. B) Aumento da espessura do corte para $3 \mathrm{~mm}$, permitindo a identificação do ponto PC e PI. C) Mensuração realizada.

sobre as radiografias (Fig. 4B, C).

Uma vez adquiridas as imagens tomográficas, as mensurações foram procedidas com a ferramenta distance do software XoranCat. No ambiente de avaliação multiplanar, na janela de exibição axial, a imagem foi rotacionada de forma que o plano oclusal do lado de interesse permanecesse orientado paralelamente ao plano vertical (Fig. 5C). Adicionalmente, na janela sagital, a imagem foi rotacionada de forma que o longo eixo do dente permanecesse perpendicular ao plano horizontal (Fig. 5B).

Os pontos foram identificados nos três planos e, por meio da ferramenta de medida disponível no programa, foram feitas as mensurações no plano coronal. Visto que a tomografia computadorizada permite uma visão tridimensional, optou-se por fazer as medidas no plano coronal, pois este permite melhor visualização dos pontos, comparado ao plano sagital. A espessura do corte tomográfico ideal para identificar o centro dos pontos de guta-percha foi de $0,2 \mathrm{~mm}$ (Fig. 6A). Porém, na maioria dos casos, os pontos não estavam no mesmo plano coronal neste corte e, para identificá-los e mensurá-los, foi necessário aumentar a espessura do corte (Fig. 6B, C). As mensurações neste grupo foram realizadas apenas uma vez, visto que foram feitas de forma digital, através do programa descrito anteriormente.

\section{Análise estatística}

Os dados foram tabulados e analisados através da estatística descritiva da variável leitura, calculando as medidas de tendência central e dispersão, avaliando a calibração e a reprodutibilidade do pesquisador. Foi empregado o teste $t$ de Student para amostras pareadas, com intervalo de confiança de 95\%, com o objetivo de comparar os exames radiográficos isoladamente com os dados do padrão-ouro.

\section{RESULTADOS}

Através das análises das medidas de tendência central e dispersão, verificou-se correlação significativa para as três leituras das radiografias interproximais e periapicais e das moldeiras de acetato, confirmando a reprodutibilidade e a calibração da variável leitura. Identificou-se p > 0,98 e p > 0,97 para o padrão-ouro, $\mathrm{p}>0,97$ e $\mathrm{p}>0,96$ para as radiografias interproximais, $\mathrm{p}>0,98$ e $\mathrm{p}>0,97$ para as radiografias periapicais, nas medidas 1 e 2 , respectivamente.

Na medida 1 foi observada diferença estatisticamente significante em relação ao padrão-ouro em $4,1 \%, 25 \%$ e $100 \%$ das áreas, nas tomografias computadorizadas de feixe cônico, nas radiografias interproximais e nas radiografias periapicais, respectivamente (Tab. 1).

Na medida 2 foi observada diferença estatisti- 
Tabela 1 - Média e desvio-padrão das três leituras da medida 1, em milímetros.

\begin{tabular}{|c|c|c|c|c|c|c|c|c|}
\hline & \multicolumn{2}{|c|}{ padrão-ouro } & \multicolumn{2}{|c|}{ interproximal } & \multicolumn{2}{|c|}{ periapical } & \multicolumn{2}{|c|}{ tomografia } \\
\hline & $\mathbf{x}$ & d.p. & $\mathbf{X}$ & d.p. & 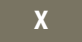 & d.p. & $\mathbf{X}$ & d.p. \\
\hline \multicolumn{9}{|l|}{$14-15$} \\
\hline paciente 1 & 6,74 & 0,12 & 6,64 & 0,02 & $4,38^{* *}$ & 0,03 & 6,55 & 0,10 \\
\hline paciente 2 & 7,63 & 0,03 & 7,46 & 0,02 & $5,35^{* *}$ & 0,03 & 7,60 & 0,10 \\
\hline paciente 3 & 8,47 & 0,05 & $8,04^{*}$ & 0,05 & $5,54^{* *}$ & 0,04 & 8,33 & 0,10 \\
\hline paciente 4 & 8,07 & 0,04 & 7,83 & 0,01 & $4,95^{* *}$ & 0,05 & 7,92 & 0,10 \\
\hline \multicolumn{9}{|l|}{$15-16$} \\
\hline paciente 1 & 6,42 & 0,07 & 6,34 & 0,04 & $3,8^{* *}$ & 0,01 & 6,17 * & 0,10 \\
\hline paciente 2 & 7,83 & 0,02 & $7,45^{*}$ & 0,03 & $5,96^{* *}$ & 0,04 & 7,83 & 0,10 \\
\hline paciente 3 & 8,01 & 0,05 & 7,94 & 0,03 & $5,44^{* *}$ & 0,01 & 7,98 & 0,10 \\
\hline paciente 4 & 8,66 & 0,02 & $7,81^{* *}$ & 0,06 & $6,25^{* *}$ & 0,12 & 8,79 & 0,10 \\
\hline \multicolumn{9}{|l|}{$24-25$} \\
\hline paciente 1 & 8,24 & 0,03 & $7,67^{* *}$ & 0,02 & $5,55^{* *}$ & 0,03 & 8,09 & 0,10 \\
\hline paciente 2 & 8,08 & 0,07 & 7,89 & 0,04 & $6,32^{* *}$ & 0,03 & 7,97 & 0,10 \\
\hline paciente 3 & 8,98 & 0,01 & $8,35^{* *}$ & 0,01 & $6,12^{* *}$ & 0,02 & 8,83 & 0,10 \\
\hline paciente 4 & 7,95 & 0,03 & 8,08 & 0,04 & $6,33^{* *}$ & 0,09 & 8,09 & 0,10 \\
\hline \multicolumn{9}{|l|}{$25-26$} \\
\hline paciente 1 & 7,88 & 0,16 & $7,02^{*}$ & 0,05 & $5,92^{* *}$ & 0,07 & 7,94 & 0,10 \\
\hline paciente 2 & 8,45 & 0,01 & 8,56 & 0,07 & $7,56^{* *}$ & 0,06 & 8,29 & 0,10 \\
\hline paciente 3 & 8,53 & 0,03 & 8,56 & 0,02 & $7,14^{* *}$ & 0,04 & 8,36 & 0,10 \\
\hline paciente 4 & 7,62 & 0,05 & 7,83 & 0,01 & $6,20^{* *}$ & 0,02 & 7,81 & 0,10 \\
\hline \multicolumn{9}{|l|}{$34-35$} \\
\hline paciente 1 & 6,53 & 0,08 & 6,31 & 0,06 & $4,90^{* *}$ & 0,02 & 6,40 & 0,10 \\
\hline paciente 2 & 8,28 & 0,03 & 7,05 & 0,03 & $7,05^{* *}$ & 0,03 & 7,99 & 0,10 \\
\hline paciente 3 & 8,26 & 0,04 & $7,75^{* *}$ & 0,04 & $6,23^{* *}$ & 0,06 & 8,41 & 0,10 \\
\hline paciente 4 & 8,99 & 0,04 & 8,98 & 0,02 & $6,72^{* *}$ & 0,02 & 8,94 & 0,10 \\
\hline \multicolumn{9}{|l|}{$35-36$} \\
\hline paciente 1 & 7,30 & 0,07 & $5,50^{* *}$ & 0,03 & $5,38^{* *}$ & 0,02 & 7,28 & 0,10 \\
\hline paciente 2 & 8,24 & 0,03 & 8,16 & 0,03 & $5,74^{* *}$ & 0,05 & 8,12 & 0,10 \\
\hline paciente 3 & 7,61 & 0,06 & 7,55 & 0,03 & $6,09^{* *}$ & 0,04 & 7,59 & 0,10 \\
\hline paciente 4 & 8,58 & 0,04 & 8,66 & 0,07 & $5,42^{* *}$ & 0,02 & 8,32 & 0,10 \\
\hline \multicolumn{9}{|l|}{$44-45$} \\
\hline paciente 1 & 7,69 & 0,03 & 7,66 & 0,03 & $5,53^{* *}$ & 0,02 & 7,52 & 0,10 \\
\hline paciente 2 & 8,99 & 0,05 & 8,84 & 0,03 & $7,36^{* *}$ & 0,03 & 9,02 & 0,10 \\
\hline paciente 3 & 7,51 & 0,03 & 7,56 & 0,01 & $6,22^{* *}$ & 0,01 & 7,57 & 0,10 \\
\hline paciente 4 & 7,70 & 0,03 & 7,64 & 0,02 & $5,91^{* *}$ & 0,05 & 7,53 & 0,10 \\
\hline \multicolumn{9}{|l|}{$45-46$} \\
\hline paciente 1 & 7,17 & 0,01 & 6,99 & 0,07 & $4,74^{* *}$ & 0,05 & 7,10 & 0,10 \\
\hline paciente 2 & 7,69 & 0,02 & 7,77 & 0,06 & $6,85^{* *}$ & 0,02 & 7,57 & 0,10 \\
\hline paciente 3 & 6,33 & 0,05 & 6,14 & 0,04 & $4,96^{* *}$ & 0,03 & 6,40 & 0,10 \\
\hline paciente 4 & 7,38 & 0,03 & 7,43 & 0,04 & $4,49^{* *}$ & 0,06 & 7,43 & 0,10 \\
\hline
\end{tabular}

Teste t de Student comparando cada área com o padrão-ouro, onde $\left(^{*}\right)$ refere-se a $p<0,05$ e ${ }^{* *}$ ) refere-se a $p<0,00$. 
Tabela 2 - Média e desvio-padrão das três leituras da medida 2, em milímetros.

\begin{tabular}{|c|c|c|c|c|c|c|c|c|}
\hline & \multicolumn{2}{|c|}{ padrão-ouro } & \multicolumn{2}{|c|}{ interproximal } & \multicolumn{2}{|c|}{ periapical } & \multicolumn{2}{|c|}{ tomografia } \\
\hline & $x$ & d.p. & $X$ & d.p. & $x$ & d.p. & $x$ & d.p. \\
\hline \multicolumn{9}{|l|}{$14-15$} \\
\hline paciente 1 & 6,74 & 0,12 & 6,56 & 0,07 & $2,50^{* *}$ & 0,04 & 6,55 & 0,10 \\
\hline paciente 2 & 7,63 & 0,03 & 7,45 & 0,07 & $4,56^{* *}$ & 1,16 & 7,60 & 0,10 \\
\hline paciente 3 & 8,47 & 0,05 & $7,04^{* *}$ & 0,63 & $4,14^{* *}$ & 0,02 & 8,33 & 0,10 \\
\hline paciente 4 & 8,07 & 0,04 & $7,65^{*}$ & 0,04 & $4,14^{* *}$ & 0,12 & 7,92 & 0,10 \\
\hline \multicolumn{9}{|l|}{$15-16$} \\
\hline paciente 1 & 6,42 & 0,07 & $5,82^{*}$ & 0,09 & $2,03^{* *}$ & 0,03 & $6,17^{*}$ & 0,10 \\
\hline paciente 2 & 7,83 & 0,02 & $7,44^{*}$ & 0,03 & $4,67^{* *}$ & 0,57 & 7,83 & 0,10 \\
\hline paciente 3 & 8,01 & 0,05 & $7,00^{* *}$ & 0,01 & $3,93^{* *}$ & 0,07 & 7,98 & 0,10 \\
\hline paciente 4 & 8,66 & 0,02 & $7,77^{* *}$ & 0,04 & $5,12^{* *}$ & 0,00 & 8,79 & 0,10 \\
\hline \multicolumn{9}{|l|}{$24-25$} \\
\hline paciente 1 & 8,24 & 0,03 & $7,50^{* *}$ & 0,01 & $3,64^{* *}$ & 0,08 & 8,09 & 0,10 \\
\hline paciente 2 & 8,08 & 0,07 & $7,65^{*}$ & 0,04 & $4,20^{* *}$ & 0,03 & 7,97 & 0,10 \\
\hline paciente 3 & 8,98 & 0,01 & $7,79^{* *}$ & 0,01 & $5,10^{* *}$ & 0,02 & 8,83 & 0,10 \\
\hline paciente 4 & 7,95 & 0,03 & 8,08 & 0,01 & $5,60^{* *}$ & 0,08 & 8,09 & 0,10 \\
\hline \multicolumn{9}{|l|}{$25-26$} \\
\hline paciente 1 & 7,88 & 0,16 & $7,00^{* *}$ & 0,01 & $4,63^{* *}$ & 0,03 & 7,94 & 0,10 \\
\hline paciente 2 & 8,45 & 0,01 & 8,53 & 0,07 & $5,95^{* *}$ & 0,07 & 8,29 & 0,10 \\
\hline paciente 3 & 8,53 & 0,03 & $7,24^{* *}$ & 0,08 & $5,32^{* *}$ & 0,07 & 8,36 & 0,10 \\
\hline paciente 4 & 7,62 & 0,05 & 7,62 & 0,04 & $5,86^{* *}$ & 0,84 & 7,81 & 0,10 \\
\hline \multicolumn{9}{|l|}{$34-35$} \\
\hline paciente 1 & 6,53 & 0,08 & 6,02 & 0,07 & $3,61^{* *}$ & 0,07 & 6,40 & 0,10 \\
\hline paciente 2 & 8,28 & 0,03 & 8,15 & 0,04 & $5,48^{* *}$ & 0,05 & 7,99 & 0,10 \\
\hline paciente 3 & 8,26 & 0,04 & $7,45^{* *}$ & 0,05 & $5,16^{* *}$ & 0,01 & 8,41 & 0,10 \\
\hline paciente 4 & 8,99 & 0,04 & 8,92 & 0,02 & $4,88^{* *}$ & 0,10 & 8,94 & 0,10 \\
\hline \multicolumn{9}{|l|}{$35-36$} \\
\hline paciente 1 & 7,30 & 0,07 & $4,85^{* *}$ & 0,06 & $3,55^{* *}$ & 0,02 & 7,28 & 0,10 \\
\hline paciente 2 & 8,24 & 0,03 & 8,15 & 0,04 & $4,23^{* *}$ & 0,09 & 8,12 & 0,10 \\
\hline paciente 3 & 7,61 & 0,06 & 7,52 & 0,04 & $5,06^{* *}$ & 0,03 & 7,59 & 0,10 \\
\hline paciente 4 & 8,58 & 0,04 & 8,65 & 0,04 & $2,83^{* *}$ & 2,81 & 8,32 & 0,10 \\
\hline \multicolumn{9}{|l|}{$44-45$} \\
\hline paciente 1 & 7,69 & 0,03 & $6,55^{*}$ & 0,32 & $2,30^{* *}$ & 0,01 & 7,52 & 0,10 \\
\hline paciente 2 & 8,99 & 0,05 & $8,41^{*}$ & 0,04 & $6,72^{* *}$ & 0,01 & 9,02 & 0,10 \\
\hline paciente 3 & 7,51 & 0,03 & $7,01^{*}$ & 0,00 & $4,71^{* *}$ & 0,02 & 7,57 & 0,10 \\
\hline paciente 4 & 7,70 & 0,03 & 7,60 & 0,02 & $4,81^{* *}$ & 0,01 & 7,53 & 0,10 \\
\hline \multicolumn{9}{|l|}{$45-46$} \\
\hline paciente 1 & 7,17 & 0,01 & $6,60^{* *}$ & 0,01 & $1,97^{* *}$ & 0,04 & 7,10 & 0,10 \\
\hline paciente 2 & 7,69 & 0,02 & 7,77 & 0,07 & $5,53^{* *}$ & 0,04 & 7,57 & 0,10 \\
\hline paciente 3 & 6,33 & 0,05 & $5,91^{*}$ & 0,04 & $3,72^{* *}$ & 0,04 & 6,40 & 0,10 \\
\hline paciente 4 & 7,39 & 0,03 & 7,44 & 0,05 & $2,88^{* *}$ & 0,12 & 7,43 & 0,10 \\
\hline
\end{tabular}

Teste t de Student comparando cada área com o padrão-ouro, onde $\left(^{*}\right)$ refere-se a $p<0,05$ e ${ }^{* *}$ ) refere-se a $p<0,00$. 
camente significante em relação ao padrão-ouro em 4,1\%, 56,2\% e 100\% das áreas, nas tomografias computadorizadas de feixe cônico, nas radiografias interproximais e nas radiografias periapicais, respectivamente (Tab. 2).

Para as radiografias interproximais, nas medidas 1 e 2, 65,3\% e 34,6\% das áreas corresponderam ao arco superior e inferior, respectivamente (Tab. 1, 2).

\section{DISCUSSÃO}

Os mini-implantes têm evoluído bastante em relação à forma, ao tipo e à técnica operatória, tornando-se recurso cada vez mais seguros e padronizados. Porém, poucos estudos têm sido feitos sobre as técnicas radiográficas disponíveis para orientação do profissional no momento do planejamento e da instalação.

No presente trabalho, as mensurações das radiografias foram realizadas sobre um papel de acetato, utilizando-se um paquímetro digital com precisão de centésimos de milímetro e não diretamente sobre a radiografia, pois a ponta do paquímetro riscaria o filme, viciando as mensurações das leituras subseqüentes. Foram realizadas três leituras de cada exame, com uma semana de intervalo entre elas, tendo como objetivo calcular a média entre as medidas. Da análise das medidas de tendência central e dispersão, observou-se correlação significativa nas três leituras. Metodologia similar foi utilizada nos estudos de Gher e Richardson ${ }^{10}$ para avaliar a reprodutibilidade e a calibração do pesquisador na realização das leituras.

Como na análise das imagens radiográficas foi observada distorção vertical e deslocamento dos pontos, foram realizadas duas medidas (medida 1 : PC - PI; medida 2: PCX - PI), pois apenas a medida 1 poderia não revelar a distorção radiográfica real.

As distorções verticais baseiam-se na profundidade em que está o objeto - neste caso, foram dois objetos, o processo alveolar e o marcador - em razão da distância para o filme. Tem-se, portanto, objetos em planos diferentes, gerando, conseqüentemente, distorções diferentes. Porém, Ruschel et al. ${ }^{25}$ recomendam, mesmo em estudo em crânios secos, que o marcador não esteja em contato com a crista óssea, para simular uma situação clínica, pois, em boca, temos o tecido gengival entre o marcador e o osso. Caso o marcador ficasse em contato com a crista óssea, teríamos dois objetos na mesma distância, porém sem estar representando uma situação clínica.

As medidas, estimadas para menos, mostram, de forma incorreta, que o mini-implante seria instalado muito próximo da crista óssea, o que contra-indicaria tal sítio, devido à possibilidade de fratura da crista óssea ou ao risco de instalação do mini-implante em tecido mole. O deslocamento do ponto mais para cervical e conseqüente diminuição da distância horizontal interdentária, devido à forma cônica das raízes, poderia levar à eleição incorreta de um mini-implante de menor diâmetro, e mesmo sugerir que o espaço observado seria inadequado para a instalação de um dispositivo temporário de ancoragem. Isso é relevante, pois, segundo Araújo ${ }^{1}$, deve-se considerar a área disponível para instalação, pois espaços reduzidos exigem mini-implantes de menor calibre. Entre as raízes, desde que exista espaço, é preferível eleger o de, no mínimo, $1,4 \mathrm{~mm}$, pois os menores apresentam maior risco de fratura quando da instalação ou remoção, principalmente na mandíbula, onde a cortical é mais espessa. Caso este espaço não seja adequado, há necessidade de se avaliar a possibilidade de utilizar áreas alternativas, modificar a angulação de instalação ou, ainda, promover ortodonticamente o afastamento das raízes, de forma a aumentar o espaço para que a fixação do mini-implante seja realizada com segurança.

Os resultados do presente estudo mostraram que as radiografias periapicais apresentam diferença estatisticamente significante em $100 \%$ das áreas (Tab. 1, 2), sendo, portanto, contra-indicadas para análise do local eleito para instalação de mini-implantes, uma vez que a imagem do guia 
estará muito deslocada em direção à crista óssea alveolar. A distorção vertical é tão expressiva que inviabiliza, inclusive, mensurações horizontais no local proposto. Adicionalmente, pode-se inferir que, como as imagens dos guias cirúrgicos sofrem grande distorção vertical nas radiografias periapicais, estas também não estariam indicadas para avaliação dos mini-implantes instalados.

$\mathrm{Na}$ medida 1, as radiografias interproximas mostraram resultados satisfatórios (Tab. 1). Apesar de apresentarem diferença estatisticamente significante em $25 \%$ das áreas, esta diferença não tem relevância clínica. Segundo Callegari-Jacques ${ }^{4}$, uma diferença estatisticamente significante encontrada em medições de imagens radiográficas em Odontologia pode não ser relevante do ponto de vista clínico ou biológico, em decorrência dos valores mensurados serem reduzidos.

Na medida 2, a radiografia interproximal apresentou diferença estatisticamente significante em 56,2\% das regiões (Tab. 2), com medidas subestimadas em relação ao padrão-ouro. $\mathrm{O}$ aumento das áreas que apresentaram diferença estatisticamente significante, da medida 1 para a medida 2, pode ser justificado pela correção do ponto de contato, que sofreu deslocamento vertical no sentido cervical, o que o aproximou mais do ponto de inserção na radiografia, exacerbando a distorção provocada

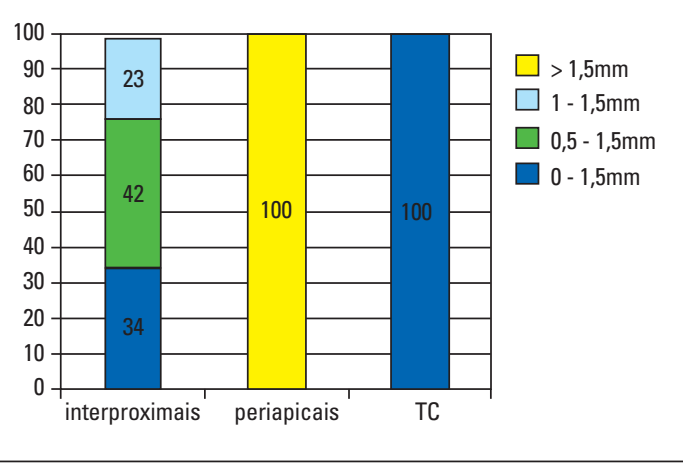

GRÁFICO 1 - Áreas que apresentaram diferença estatisticamente significante em relação ao padrão-ouro, distribuídas de acordo com o intervalo de milímetros. pela angulação vertical.

Observa-se, nas radiografias interproximais, que o arco superior apresentou maior distorção em relação ao arco inferior, o que pode ser explicado pela angulação vertical positiva utilizada no procedimento $\left(+5^{\circ} \mathrm{a}+10^{\circ}\right)$, mais adequada para o arco inferior, devido à implantação dos dentes na base óssea. Analisando as áreas que apresentaram diferença estatisticamente significante em relação ao padrão-ouro, observa-se que 34,6\%, 42,3\% e $23 \%$ apresentaram distorção vertical na faixa de 0 a $0,5 \mathrm{~mm}, 0,5$ a $1 \mathrm{~mm}$ e 1 a $1,5 \mathrm{~mm}$, respectivamente (Gráf. 1). Apesar da baixa porcentagem de regiões na faixa entre 1,0 e 1,5mm, deve-se ter cautela em utilizar as radiografias interproximais com os propósitos citados nesta pesquisa, pois, não raro, o espaço inter-radicular é restrito, e 1,5mm pode ter relevância clínica.

$\mathrm{Na}$ comparação dos resultados entre as radiografias intrabucais, constata-se que as interproximais são mais precisas que as periapicais. Estes resultados estão em consonância com os de Reed e Polson ${ }^{24}$, Sewerin ${ }^{27}$ e Thunthy ${ }^{29}$, que relataram que, quando a ancoragem com mini-implantes for necessária, o local eleito deverá ser avaliado com radiografia interproximal, pois as periapicais produzem imagens obliquamente projetadas e distorcidas.

A tomografia computadorizada de feixe cônico apresentou resultados estatisticamente significantes em apenas $4,1 \%$ da amostra, com diferença de, no máximo, $0,50 \mathrm{~mm}$, sendo assim considerado o método mais preciso e mais fiel para visualização dos sítios propostos para instalação de mini-implantes.

Para pacientes iniciais, o profissional deverá avaliar cuidadosamente a relação custo-benefício e solicitar a tomografia computadorizada de feixe cônico, que oferecerá, em apenas um exame, todas as imagens convencionais em 2D que compõem a documentação ortodôntica, somadas à visão tridimensional detalhada das estruturas dentofaciais. Sob a perspectiva do custo financeiro, a tomografia 
computadorizada mostra-se muito compensadora, pois, atualmente, o orçamento do exame equivale, aproximadamente, ao da documentação convencional de Ortodontia. Quanto ao custo biológico, vinculado à exposição do paciente à radiação, deve-se considerar que o exame de tomografia computadorizada de feixe cônico pode substituir diversas tomadas radiográficas convencionais utilizadas como rotina na Ortodontia ${ }^{9}$.

\section{CONCLUSÃO}

Levando em consideração os resultados apresentados, conclui-se que a tomografia computadorizada de feixe cônico foi o exame mais preciso e efetivo para avaliar a posição vertical dos sítios eleitos para instalação de mini-implantes, com di- ferenças em relação ao padrão-ouro não superiores a $0,50 \mathrm{~mm}$. A radiografia interproximal pode ser utilizada com cautela e a radiografia periapical apresentou resultados insatisfatórios.

\section{AGRADECIMENTOS}

À Odonto Bio Imagem e aos Drs. João Carlos Costa da Silva e Elmo Anísio Costa da Silva pela inestimável contribuição para a realização deste trabalho.

\title{
The accuracy of radiographic techniques used for vertical localization of mini-implants fixture placement
}

\begin{abstract}
Objective: Evaluate the effectiveness of image diagnosis methods used in the vertical location of sites elect to insert mini-implants. Methods: The sample was composed of four patients, in which 32 inter-radicular sites were established in the subsequent area, for setting up mini-implants. These sites were represented by holes filled out with guta-percha in acetate moulder (PCg - contact point of dental crowns in the acetate moulder; Plg - point of insertion of the mini-implant in the acetate moulder). Periapical X-rays, interproximal and computerized tomographies of cone-beam, with the moulders positioned in mouth, were performed and the following points were taken into consideration: PC - radiodense image of the PCg point; PI - radiodense image of the Plg point; PCx - contact point among the determined dental crowns on the X-ray. Vertical measures used: gold-pattern from PCg to Plg; measure 1 - from PC to Pl; and measure 2 - from PCx to PI. The measures were compared through descriptive analysis and test $t$ of Student. Results: For the measure 1, differences statistically significant were observed (4.1\%), in relation to the gold-pattern, $25 \%$ and $100 \%$ of the appraised measures in the computerized tomographies, interproximal and periapical X-rays, respectively. For the measure 2, differences statistically significant were observed in relation to the gold-pattern (4.1\%), $56.2 \%$ and $100 \%$ of the appraised measures in the computerized tomographies, interproximal and periapical X-rays, respectively Conclusions: The cone-beam computerized tomography was the most precise exam in the evaluation of the vertical position of the elect sites to install mini-implants; the interproximal X-ray can, with reservation, be also used; the periapical X-ray presented unsatisfactory results, being contraindicated for this purpose.
\end{abstract}

Key words: Diagnosis by image. Dental X-ray. X-ray computerized tomography. Orthodontic anchorage procedures. 


\section{REFERÊNCIAS}

1. ARAÚJO, T. M. Ancoragem esquelética com mini-implantes. In: LIMA FILHO, R. M. A. BOLOGNESE, A. M. Ortodontia: arte e ciência, 1. ed. Maringá: Dental Press, 2007. p. 393-448.

2. ARAÚJO, T. M.; NASCIMENTO, M. H. A.; BEZERRA, F.; SOBRAL, M. C. Ancoragem esquelética em Ortodontia com mini-implantes. Rev. Dental. Press Ortodon. Ortop. Facial, Maringá, v. 11, n. 4, p. 126-156, jul./ago. 2006.

3. BAE, S. M.; PARK, H. S.; KYUNG, H. M.; KWON, O. W. SUNG, J. H. Clinical application of micro-implant anchorage. J. Clin. Orthod., Boulder, v. 36, no. 5, p. 298-302, May 2002.

4. CALLEGARI-JACQUES, S. M. Bioestatística: princípios e aplicações. Porto Alegre: Artmed, 2003. p. 247-258.

5. COSTA, A.; RAFFAINL, M.; MELSEN, B. Miniscrews as orthodontic anchorage: a preliminary report. Int. J. Adult Orthodon. Orthognath. Surg., Chicago, v. 13, no. 3, p. 201-209, 1998.

6. DUCKWORTH, J. E.; JUDY, P. F.; GOODSON, J. M. SOCRANSKY, S. S. A method for the geometric and densitometric standardization of intraoral radiographs. J. Periodontol., Chicago, v. 54, no. 7, p. 435-440, July 1983.

7. FABBRONI, G.; AABED, S.; MIZEN, K.; STARR, D. G. Transalveolar screws and the incidence of dental damage: a prospective study. Int. J. Oral Maxillofac. Surg. Copenhagen, v. 33, no. 5, p. 442-446, July 2004.

8. FREITAS, A.; VAROLI, O. J.; TORRES, F. A. Técnicas radiográficas intrabucais. In: FREITAS, A.; ROSA, J. E. SOUZA, I. F. Radiologia odontológica. 1. ed. São Paulo: Artes Médicas, 2004. p. 103-166.

9. GARIB, D. G.; RAYMUNDO JUNIOR, R.; RAYMUNDO, M. V.; RAYMUNDO, D. V.; FERREIRA, S. N. Tomografia computadorizada de feixe cônico (Cone beam): entendendo este novo método de diagnóstico por imagem com promissora aplicabilidade na Ortodontia. Rev. Dental Press Ortodon. Ortop. Facial, Maringá, v. 12, n. 2, p. 139-156, mar./apr. 2007

10. GHER, M. E.; RICHARDSON, A. C. The accuracy of dental radiographic techniques used for evaluation of implant fixture placement. Int. J. Periodontics Restorative Dent., Chicago, v. 15 , no. 3, p. 268-283, June 1995

11. GRABER, T. M.; VANARSDALL, R. L. Orthodontics: current principles and techniques. 5th ed. Missouri: Elsevier, 2005.

12. KANOMI, R. Mini-implant for orthodontic anchorage. J. Clin. Orthod., Boulder, v. 31, no. 11, p. 763-767, Nov. 1997.

13. KIM, S. H.; CHOI Y. S.; HWANG, E. H.; CHUNG, K. R.; KOOK, Y. A.; NELSON, G. Surgical positioning of orthodontic mini-implants with guides fabricated on models replicated with cone-beam computed tomography. Am. J. Orthod. Dentofacial Orthop., St. Louis, v. 131, no. 4, p. S82-S89, Apr. 2007. Supplement.

14. KITAI, N.; YASUDA, Y.; TAKADA, K. A stent fabricated on a selectively colored stereolithographic model for placement of orthodontic mini-implants. Int. J. Adult Orthodon.

Orthognath. Surg., Chicago, v. 17, no. 4, p. 264-266, 2002.
15. KOYANAGI, K. Development and clinical application of a surgical guide for optimal implant placement. J. Prosthet. Dent., St. Louis, v. 88, no. 5, p. 548-552, Nov. 2002.

16. KURODA, S.; YAMADA, K.; DEGUCHI, T.; HASHIMOTO, T.; KYUNG, H. M.; TAKANO-YAMAMOTO, T. Root proximity is a major factor for screw failure in orthodontic anchorage. Am. J. Orthod. Dentofacial Orthop., St. Louis, v. 131, no. 4, p. S68-S73, Apr. 2007. Supplement.

17. KYUNG, S. H.; CHOI, J. H.; PARK, Y. C. Miniscrew anchorage used to protract lower second molars into first molar extraction sites. J. Clin. Orthod., Boulder, v. 37, no. 10, p. 575-779, Oct. 2003.

18. KYUNG, H. M.; PARK, H. S.; BAE, S. M.; SUNG, J. H.; KIM, I. B. Development of orthodontic micro-implants for intraoral anchorage. J. Clin. Orthod., Boulder, v. 37, no. 6, p. 321-328, June 2003.

19. MAH, J.; BERGSTRAND, F. Temporary anchorage devices: a status report. J. Clin. Orthod., Boulder, v. 39, no. 3, p. 132-136, Mar. 2005

20. MAINO, B. G.; BEDNAR, J.; PAGIN P.; MURA, P. The spider screw for skeletal anchorage. J. Clin. Orthod., Boulder, v. 37, no. 2, p. 90-97, Feb. 2003

21. MOREA, C.; DOMINGUEZ, G. C.; WUO, A. V.; TORTAMANO, A. Surgical guide for optimal positioning of mini-implants. J. Clin. Orthod., Boulder, v. 39, no. 5 p. 317-321, May 2005

22. PARK, H. S.; BAE, S. M.; KYUNG, H. M.; SUNG, J. H.; Microimplant anchorage for treatment of skeletal Class I bialveolar protrusion. J. Clin. Orthod., Boulder, v. 35, no. 7, p. 417-422, July 2001.

23. POGGIO, P. M.; INCORVATI, C.; VELO, S.; CARANO, A. "Safe zones": a guide for miniscrew positioning in the maxillary and mandibular arch. Angle Orthod., Appleton, v. 76, no. 2, p. 191-197, Mar. 2006

24. REED, B. E.; POLSON, A. M. Relationships between bitewing and periapical radiographs in assessing crestal alveolar bone levels. J. Periodontol., Chicago, v. 55, no. 1, p. 22-27, Jan. 1984

25. RUSCHEL, G.; NACONECY, M. M.; VEECK, E. B.; COSTA, N. P. Tomografia linear $\mathrm{X}$ tomografia computadorizada. Rev. Odonto Cienc., Porto Alegre, v. 16, n. 34, p. 264-267, set./dez. 2001

26. SCHNELLE, M. A.; BECK, F. M.; JAYNES, R. M.; HUJA, S. $S$. A radiographic evaluation of the availability of bone for placement of miniscrews. Angle Orthod., Appleton, v. 74, no. 6, p. 832-837, Dec. 2004.

27. SEWERIN, P. Utilize your dental X-ray set better. Int. Dent. J., London, v. 37, no. 1, p. 38-42, Mar. 1987.

28. SUZUKI, E. Y.; BURANASTIDPORN, B. An adjustable surgical guide for miniscrew placement. J. Clin. Orthod., Boulder, v. 39, no. 10, p. 588-590, Oct. 2005.

29. THUNTHY, K. H. Radiographic illusions due to faulty angulations. Dent. Radiogr. Photogr., Rochester, v. 51, no. 1, p. 1-7, 13-15, 1978.
Endereço para correspondência

Liz Matzenbacher da Silva

Centro de Ortodontia e Ortopedia Facial

AV. Araújo Pinho, $n^{\circ}$ 62, $7^{\circ}$ andar - Canela - Salvador / BA

CEP: 40.110-150 - E-mail: lizmdasilva@hotmail.com 